\title{
Ist Agilität Voraussetzung oder Folge einer zielgerichteten Digitalisierung?
}

\author{
Dominic Lindner und Michael Amberg, FAU Erlangen-Nürnberg - \\ Lehrstuhl für Wirtschaftsinformatik III inbs. IT-Management
}

\begin{abstract}
Agility as Consequence or Prerequisite of Digitization?

Companies have always been in a constant state of change. This change is today closely linked to the buzzword's "digitization" and "agility". Agile methods, especially in complex projects, can pave the way for targeted digitization and, on the other hand, provide a more agile way of working for digital technologies. Through group discussions with managers from small and medium-sized IT companies, this article focuses on the question of whether agility is the precondition or consequence of targeted digitization. This article is aimed at decision-makers from SMEs who want to increase the degree of agility in the company in the context of increasing digitization.

Keywords:

Agility, Digization, Digitalization, SME, Management
\end{abstract}

\begin{abstract}
Unternehmen befinden sich schon immer in einen konstanten Wandel, welcher heutzutage u. a. eng mit den Schlagworten „Digitalisierung“ und „Agilität" verknüpft ist. Dabei können agile Methoden speziell in komplexen Projekten Wegbereiter für eine zielgerichtete Digitalisierung sein und andererseits digitale Technologien eine agilere Arbeitsweise fördern. Dieser Beitrag fokussiert durch Gruppendiskussionen mit Managern aus mittelständischen IT-Unternehmen die Frage, ob Agilität Voraussetzung oder Folge einer zielgerichteten Digitalisierung ist. Dieser Beitrag richtet sich an Entscheider aus dem IT-Mittelstand, welche den Grad an Agilität im Unternehmen im Kontext einer zunehmenden Digitalisierung erhöhen möchten.
\end{abstract}

Die Begriffe „Digitalisierung" und "Agilität" sind aktuell populäre Schlagworte in der gesamten deutschen Unternehmenslandschaft, die vorwiegend durch mittelständische Unternehmen dominiert wird. Diese sind aufgrund ihrer Innovationsfähigkeit und Erfahrung eine wichtige Stütze der deutschen Wirtschaft [1]. Dabei sind viele dieser Unternehmen oft noch klassisch organisiert und auf Steuerbarkeit und Kontrolle optimiert, was in einem Widerspruch zu den fundamentalen Änderungen und der hohen Geschwindigkeit des digitalen Wandels steht [2].

\section{Der Mittelstand im Spannungsfeld zwischen Agilität und Digitalisierung}

Über die Entwicklung und Veränderung der Organisation von Unternehmensstrukturen und Prozessen wird schon eine lange Zeit diskutiert. Mittlerweile dominieren die Organisationsentwicklung von Unternehmen jedoch die Schlagworte Digitalisierung und Agilität mit Fokus auf Vertrauen, Selbstorganisation und Autonomie. Dabei ist ein Teil dieser Debatte die Steigerung von Agilität in Organisationen. Agilität bedeutet allgemein, die Fähigkeit eines Individuums oder Objekts, flexibel auf Anforderungen zu reagieren und sich seiner Umwelt anzupassen [3]. Somit könnte eine gesteigerte Agilität es Unternehmen ermöglichen, komplexe und anspruchsvolle Digitalisierungsprojekte mithilfe von agilen Methoden zielführender umzusetzen. Andererseits wird jedoch auch diskutiert, ob nicht die Digitalisierung eine Voraussetzung für eine gesteigerte Agilität darstellt. So können beispielsweise Tools wie Jira [4], Trello [5], Slack [6] Chatsysteme, Cloud-Software sowie mobile Hardware wie Laptops und Smartphones eine verteilte und agile Arbeit fördern.

In zahlreichen Gesprächen mit Entscheidern aus dem IT-Mittelstand haben wir bemerkt, dass aktuell nicht klar ist, wie beide Begriffe in Zusammenhang stehen. In Vorgesprächen mit Experten aus dem IT-Mittelstand wurde wiederholt explizit die folgende Frage gestellt: Sollte zuerst Agilität im Unternehmen gesteigert werden oder ist eine zielgerichtete Digitalisierung Voraussetzung für eine höhere Agilität im Unternehmen?

Zur Beantwortung dieser Frage wurde gemeinsam mit Vorständen und Geschäftsführern von mittelständischen Unternehmen aber auch Konzernen sowie Vorständen von Gewerkschaften eine Gruppendiskussion (Bild 1) zur Evaluation der beiden Thesen durchgeführt [3]. In einer zweiten Gruppendiskussion wurden mit zeitlichem Abstand mit dem nahezu gleichen Teilnehmerkreis Handlungsempfehlungen sowie ein Modell zur Agilitätssteigerung im Kontext der Digitalisierung konzipiert. Die Originalstudie [3] wurde am Lehrstuhl für IT-Management der FAU Erlangen-Nürnberg durchgeführt.

\section{Agilität als Folge der Digitalisierung}

Die Digitalisierung kann der Wegbereiter für eine gesteigerte Agilität in Organisationen sein. dominic.lindner@agileunternehmen.de www.agile-unternehmen.de 
Ein Beispiel dafür ist beispielsweise die Vision der NewWork: „Arbeite wann, wie und wo du willst". Diese geforderte Orts- und Zeitflexibilisierung von Arbeit kann mit standardisierten Desktop-Computern und klassischer Software kaum umgesetzt werden. Auch die Arbeit mit verteilten Teams ist ohne ausreichende, mobile Technologie kaum möglich [7]. Neben einer höheren Geschwindigkeit und Dynamik von Arbeit wird auch das Unternehmen als Arbeitgeber attraktiver und kann zusätzliche Fachkräfte gewinnen. Weiterhin können ebenfalls durch Software gewisse Unternehmensprozesse zielführend teil- oder sogar vollständig automatisiert werden. Beispiele solcher mobilen Technologien für eine agile Teamarbeit sind:

- Mobile Computing, Cloud Computing, Virtualisierung, Voice over IP (VoIP);

- Ubiquitärer und kollaborativer Zugriff auf Dokumente;

- Soziale Medien, Unternehmenswikis, E-Mail-Kommunikation;

- Web- und Videokonferenzen, Instant Messaging;

- Bildschirmfreigabe und virtuelle Whiteboards und

- Laptops und Smartphones.

Agilität als Vorrausetzung für die Digitalisierung

Im Gegensatz zu den gerade genannten Vorteilen der Digitalisierung liegt es jedoch auf der Hand, dass mit der Digitalisierung auch komplexe Veränderungen einhergehen. Eine davon ist beispielsweise die Einführung von Software, Prozessdigitalisierung als auch eine geforderte IT-Reorganisation, welche von Unternehmen disruptive Veränderungen fordern kann. Dies ist oft in einer eher klassischen/tayloristischen Organisation schwer umsetzbar und fordert daher agilere Strukturen und Vorgehensweise. Beispiele solcher Vorgehensweisen sind:

- Scrum [8]

- Kanban [9]

- Lean Startup [10]

- Design Thinking [11]

- DevOps [12]

Ziel ist es, komplexe Digitalisierungsprojekte mit Experimenten iterativ zu erproben und zu evaluieren, wie ein höherer Erfolg erreicht werden kann. So soll Mitarbeitern die maximale Freiheit in der Arbeitsausführung gegeben werden. Vorteile sind eine höhere Zufriedenheit der Fachkräfte als auch eine einfachere Kurskorrektur in komplexen Digitalisierungsprojekten sowie eine höhere Geschwindigkeit in stark umkämpften Märkten [7]. Die folgende Abbildung zeigt diese beiden Argumentationen auf.

\begin{tabular}{|l|l|c|}
\hline Unternehmen & Position & Anzahl MA \\
\hline Gewerkschaft & & - \\
\hline IT-Dienstleister (CRM) & Vorstand IT & 120 \\
\hline IT-Dienstleister (Logistik) & Geschäftsleitung & 400 \\
\hline IT-Dienstleister (E-Commerce) & Geschäftsleitung & 30 \\
\hline IT-Dienstleister (Rechenzentrum) & Geschäftsleitung & Vorstand \\
\hline IT-Dienstleister (Finance) & Manager & 7000 \\
\hline IT-Dienstleister (Medien) & Geschäftsleitung & 1600 \\
\hline IT-Dienstleister (Consulting) & Vorstand & 120 \\
\hline IT-Dienstleister (Industrie) & Aufsichtsrat & 200 \\
\hline
\end{tabular}

\section{Digitalisierung und Agilität im Mittel- stand}

Mittelständische Unternehmen kennzeichnen sich durch die Einheit von Eigentum und Leitung. Dabei übt der Unternehmer (oftmals Gründer) einen maßgeblichen, persönlichen Einfluss aus, trägt das unternehmerische Risiko und sichert seine persönliche Erwerbs- und Existenzgrundlage [1].

Dabei unterscheiden sich mittelständische Unternehmen oft maßgeblich von Konzernen. Für diesen Beitrag werden zwei maßgebliche Unterschiede in den Vordergrund gerückt: Grad der Digitalisierung und Grad der Agilität (Bild 2).

Auf der einen Seite verfügen mittelständische Unternehmen über weniger personelle Ressourcen sowie Budgets und verfügen deswegen oft über eine IT-Landschaft, die eher weniger komplex ausgeprägt und mehr zentralisiert ist, sodass eine Skalierung und Erweiterungen oft abwartend gesehen werden. Auf der anderen Seite gelten mittelständische Unternehmen aufgrund der geringen Prozesslandschaft, der kurzen Entscheidungswege und der Hands-on-Mentalität als deutlich flexibler und agiler als Konzerne [3].

\section{Agilität als Vorrausetzung oder Folge der Digitalisierung im Mittelstand?}

Um die Fragestellung zu erörtern, wurde eine Gruppendiskussion mit Vertretern aus dem Mittelstand durchgeführt. Aus dieser Diskussion wird abgeleitet, ob diese Digitalisierung als Voraussetzung für eine gesteigerte Agilität oder als Folge sehen.
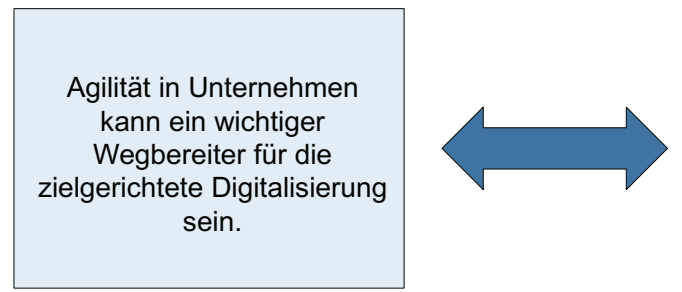

Die Einführung mobiler Hard- und Software, also eine Digitalisierung in Unternehmen, kann eine agile Arbeit fördern.
Bild 1: Teilnehmer der beiden Gruppendiskussionen [3].

Bild 2: Paradigma zwischen Agilität und Digitalisierung. 
Grad der Digitalisierung

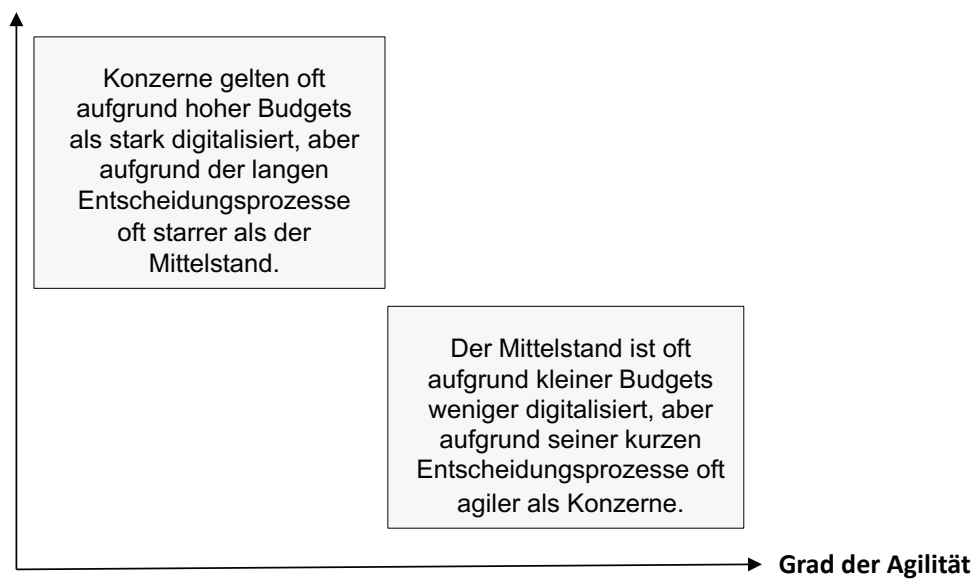

Bild 3: Paradigma zwischen Agilität und Digitalisierung in Bezug auf den IT-Mittelstand.
Der große Vorteil einer Gruppendiskussion ist, dass durch die Interaktion der Teilnehmer neues, gemeinschaftlich bestätigtes Wissen auch aus implizitem Verhalten abgeleitet werden kann. Sowohl Reaktionen (Nicken, verbale Zustimmung) als auch das Ausbleiben von Reaktionen (Kein Widerspruch, Enthaltung, Schweigen) können in der gesamten Runde einen Konsens aufzeigen. Konsens-Aussagen sind besonders wertvoll, da sie die Stimme jedes Einzelnen berücksichtigen und somit ein hohes Gewicht haben. Solche werden im Folgenden genutzt um die Frage zum Zusammenhang von Digitalisierung und Agilität zu beantworten.

Die erste Konsensaussage der Gruppendiskussion ist, dass die Digitalisierung eine wichtige Rolle als strategischer Vorteil von Unternehmen ist und agile Methoden zur Umsetzung der komplexen Digitalisierung immer wichtiger werden.

Ein weiterer Konsens der Teilnehmer ist, dass agile Methoden zusätzlich ein wichtiger Faktor der Mitarbeiterzufriedenheit, speziell in der IT, sind. Ein Grund ist laut des Vorstands der Gewerkschaft die erhöhte Flexibilität in der Arbeitsausführung. Konsens der Teilnehmer ist, dass durch strenge Prozesse und wenig Spielraum werden negative Aspekte wie Unzufriedenheit, Burn-Out und Demotivation gefördert. Auch können digitale Technologien sowie Konzepte wie Homeoffice ohne ausreichende Rahmenbedingungen nicht effizient genutzt werden.

Ähnliches bemerkt auch der Manager des IT-Dienstleister für CRM-Software. Er glaubt, dass Digitalisierungsprojekte zahlreiche neue Fachkräfte benötigen und agile Methoden im Unternehmen das Recruiting fördern. Seine Herausforderung ist, dass Mitarbeiter oft eher in den Konzernen der Region aufgrund hoher Gehälter arbeiten und er sein Unternehmen durch moderne Arbeitsbedingungen differenzieren möchte. Der Manager möchte deswegen den
Fachkräften verschiedenste Arbeitsmethoden im Unternehmen bieten und die ursprünglichen Business Units wie folgt reorganisiert [3]:

- Holacracy für die Sparte "Consulting"

- Scrum für die Sparte "hauseigene Software"

- klassische Hierarchie für die Sparte "Softwarelösungen"

Nachdem im ersten Teil der Gruppendiskussion vorrangig auf Agilität im Kontext der Mitarbeiter eingegangen worden ist, schwenken die Teilnehmer nun auf die Abwicklung von Digitalisierungsprojekten um. Der E-Commerce-Dienstleister ist ein Berater und Umsetzer in der komplexen Digitalisierung der Verkaufsprozesse seiner Kunden. Der Manager bemerkt jedoch, dass solche Projekte sehr komplex sind und in letzter Zeit zu Beschwerden führen. Beispielsweise wurden laut des Managers Kundenumfragen direkt so umgesetzt, wie der Kunden es wünschte. Leider war der Nutzen nicht so hoch wie vom Kunden gewünscht, was trotz fachgerechter Umsetzung zu geringer Kundenzufriedenheit führt. Er forderte deswegen eine proaktive Beratung und engen Kundenkontakt und etablierte die agile Methode Scrum im Unternehmen.

Ein weiteres Beispiel erläutert das Mitglied der Geschäftsleitung des IT-Logistik-Dienstleisters. Oftmals sind digitale Marketingprojekte in seinen Bereich sehr komplex und fordern Wissen aus der Logistikbranche sowie klassischer Marketingmethoden. Speziell die Beschreibung der Ziele und Aufgaben ist laut des Mitglieds der Geschäftsleitung in einem dynamischen Umfeld kaum möglich und kann mithilfe agiler Methoden sinnvoll und explorativ erprobt werden. Der Konsens der Teilnehmer ist, dass die Veränderungsprozesse/Projekte im Rahmen der Digitalisierung für eine hohe Komplexität sorgen und ohne agile Methoden oft kaum erfolgreich realisiert werden.

\section{Agilität als Vorrausetzung der Digitali- sierung im Mittelstand}

Konsens der Gruppendiskussion war, dass eine gesteigerte Agilität Voraussetzung für eine erfolgreichere Digitalisierung ist. Zusammenfassend gliedern sich die Aussagen der Gruppendiskussion in zwei Argumentationen, warum eine gesteigerte Agilität vor der Digitalisierung im Unternehmen wichtig erscheint. Es können deswegen folgende Thesen ableitet werden [3]:

These 1: Die Digitalisierungsprojekte in Unternehmen stellen zunehmend erhöhte Flexibilitätsanforderungen an Mitarbeiter in der Arbeitsausführung. Agilität kann eine höhere 
Flexibilität in der Arbeitsausführung bieten und damit Mitarbeitermotivation und Produktivität steigern. Auch werden neue Fachkräfte notwendig sein, welchen mit agilen Methoden eine attraktivere Arbeitsumgebung geschaffen werden kann.

These 2: Die Teilnehmer glauben, dass speziell Digitalisierungsprojekte eine erhöhte Komplexität bieten und auf Kundenanfragen individueller reagiert werden kann. Agile Methoden bzw. Prinzipien werden ebenfalls als eine effiziente Methode gesehen, um in Digitalisierungsprojekten flexibler und schneller reagieren zu können.

\section{Ein Modell zur Steigerung von Agilität}

Nachdem die Experten aus der ersten Gruppendiskussion der Meinung sind, dass Agilität eine Voraussetzung für eine zielgerichtete Digitalisierung ist, sollen zum Abschluss des Beitrags Empfehlungen zur Steigerung von Agilität gegeben werden. Dazu wurde ein zweiter Roundtable durchgeführt. Die Teilnehmer basieren ihre Empfehlungen auf zwei Annahmen [3]:

- Nicht jeder Mitarbeiter möchte agil arbeiten.

- Nicht jeder Bereich in Unternehmen benötigt eine hohe Agilität.

Dabei glauben die Teilnehmer, dass nicht jeder Mitarbeiter agil arbeiten möchte. Zahlreiche Mitarbeiter möchten laut den Teilnehmern der Gruppendiskussion einen Job nach Anweisungen ausführen. Der Drang vieler Mitarbeiters nach Selbstorganisation ist eher gering und die Selbstorganisation wird sogar oft als zu anstrengend empfunden. Dies kann jedoch auch ein Vorteil sein, da ein Unternehmen neben hochkreativen Bereichen mit agilen Methoden auch zahlreiche wiederholende und standardisierte Bereiche wie z.B. Elektrik und Buchhaltung benötigen.

Zusammenfassend wollen die Teilnehmer durch die Steigerung von Agilität in ausgewählten Bereichen des Unternehmens vier konkrete Ziele erreichen:

- Steigerung der Mitarbeiterzufriedenheit

- Flexibilität in der Arbeitsausführung

- Reaktion auf Veränderung

- Höhere Kundenzufriedenheit

Um die Mitarbeiterzufriedenheit zu steigern, sollen Bereiche mit verschiedener Agilität im Unternehmen geschaffen werden, sodass jeder Mitarbeiter frei wählen kann. Um die benötigen Grade an Agilität zu erreichen, empfehlen die Teilnehmer eine Aufteilung in drei Sektoren, die sich in der Nähe zum Markt unterscheiden

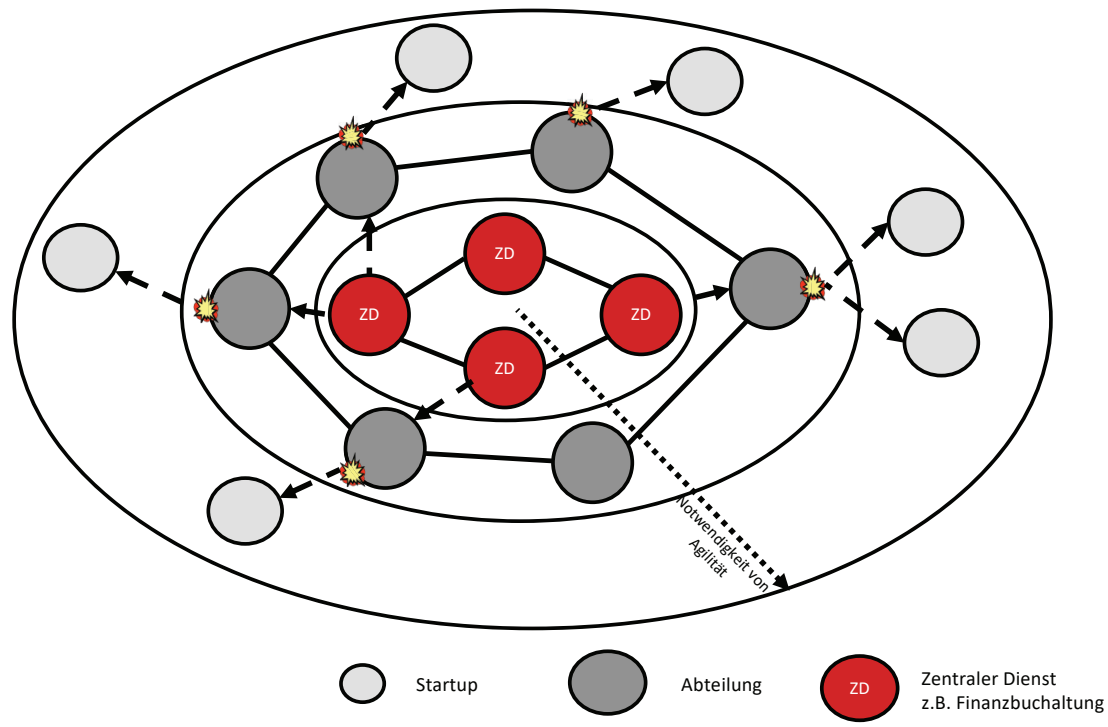

(Bild 3). So benötigen die zentralen Dienste (internes Controlling etc.) im Kreisinneren oft keinen direkten Kontakt zum Markt, größere stabile Abteilungen (bspw. stabiler und langjähriger Kunde) eine mittlere Agilität und speziell neue Pilotteams eine hohe Agilität. Ein Pilotteam (Startup) kann sich aus einer Abteilung abspalten.

Eine weitere Empfehlung der Teilnehmer sind flexible und selbstorganisierte Teams. Ziel ist eine höhere Autonomie und Flexibilität in der Arbeitserledigung. Diese Rahmenbedinungen bieten den Mitarbeitern eine höhere Freiheit um komplexe Aufgaben zu erledigen und in dynamischen Projekten schneller auf Änderungen reagieren zu können. Durch direkten Kundenkontakt können Anforderungen schneller evaluiert und Änderungswünsche mit den Kunden schnell abgestimmt werden, was zu einer höheren Kundenzufriedenheit führt. In der Zusammenfassung sehen die Teilnehmer als Grundlage einen Methodenmix im Unterneh-

Bild 4: Modell zur Steigerung von Agilität [3].

Bild 5: Neuorganisation der Abteilung und Darstellung der Teams.

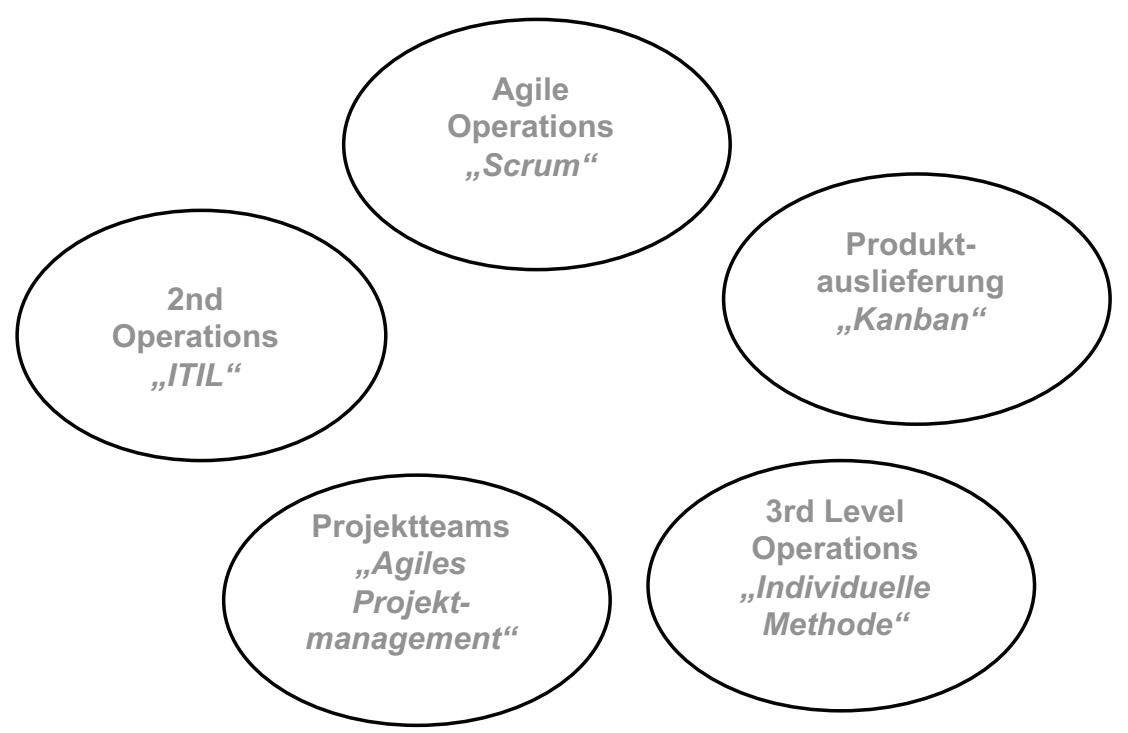




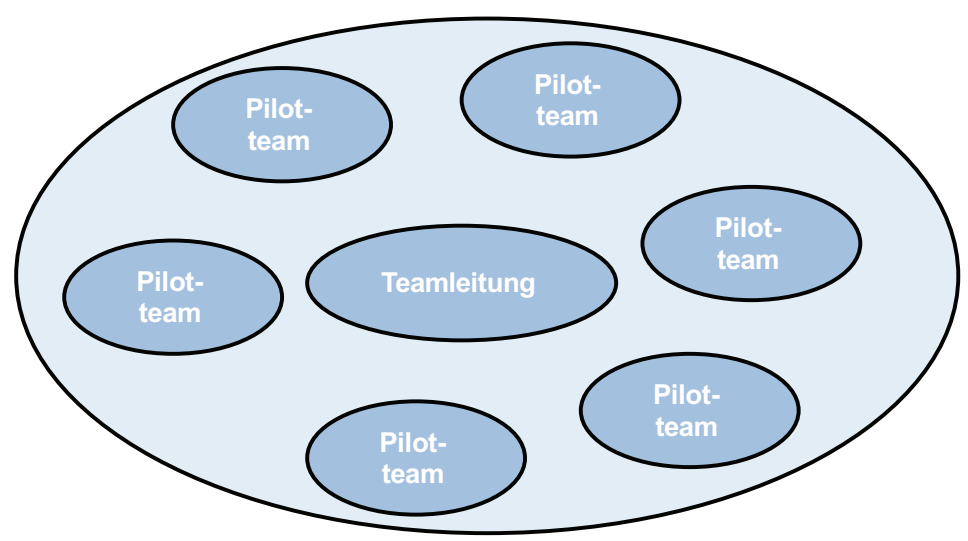

Bild 6: Handlungsempfehlungen aus der Gruppendiskussion [3].

[4] URL: https://de.atlassian.com/ software/jira, Abrufdatum 19.03.2019.

[5] URL: https://de.atlassian.com/ software/jira, Abrufdatum 19.03.2019.

[6] URL: https://slack.com/ intl/de-de, Abrufdatum 19.03.2019

[7] Lindner, D.: KMU im digitalen Wandel: Ergebnisse empirischer Studien. Wiesbaden 2019.

[8] URL: https://agile-unternehmen.de/was-ist-scrum/, Abrufdatum 20.06.2019.

[9] URL: https://agile-unternehmen.de/was-ist-kanban/, Abrufdatum 20.06.2019.

[10] URL: https://agile-unternehmen.de/lean-startup-methode Abrufdatum 20.06.2019.

[1 1] URL: https://agile-unternehmen.de/design-thinking-methode, Abrufdatum 20.06.2019

[12] URL: https://agile-unternehmen.de/devops, Abrufdatum 20.06.2019.

[13] URL: www.ifm-bonn.org/ definitionen, Abrufdatum 19.03.2019.

men aus klassischen und agilen Methoden, je nach Bereich.

\section{Beispiel zur Umsetzung der Hand- lungsempfehlungen}

Im Anschluss an dem Roundtable hat der IT-Rechenzentrumsdienstleister die Handlungsempfehlungen in einer Abteilung umgesetzt. Grundidee war verschiedene agile Methoden je nach Mitarbeiter und Zielsetzung der der Aufgabenstellung einzusetzen. Die Abteilung für den Betrieb und die Entwicklung der IT-Systeme verfügte über 70 Mitarbeiter und wurde in 5 Teams neu strukturiert. Die neuen Teams sind in der folgenden Abbildung dargestellt.

Zuerst wurde der IT-Betrieb je nach Kunden und Mitarbeiter in zwei Teams geteilt (20 Mitarbeiter pro Team). Das Team Operations bedient Kunden, welche nach ITIL klassische Standardprodukte beziehen. Das Team agile Operations dazu im Gegensatz Kunden mit dem Wunsch nach Scrum oder sehr individuellen Anforderungen an den IT-Betrieb. Um die Pilottests des Konzepts umzusetzen, wurden in den einzelnen Teams 4-8 Mitarbeiter zu losen aber fokussierten Gruppen mit gemeinsamen Ziel und gewählten Sprecher zusammengefasst (Bild 6). Diese sind oftmals einem Kunden oder Kundengruppe zugeordnet und können autonom je nach Kunden

Bild 7: Handlungsempfehlungen zur Steigerung von Agilität.

\begin{tabular}{|l|l|}
\hline Angestrebtes Ziel & Handlungsempfehlungen aus der Gruppendiskussion \\
\hline $\begin{array}{l}\text { Steigerung der } \\
\text { Mitarbeiter- } \\
\text { zufriedenheit }\end{array}$ & $\begin{array}{l}\text { Durch Bereiche mit verschiedener Agilität werden im Unternehmen } \\
\text { Möglichkeiten für jeden Mitarbeiter geschaffen, sodass dieser frei } \\
\text { wählen kann. }\end{array}$ \\
\hline $\begin{array}{l}\text { Flexibilität in der } \\
\text { Arbeitsausführung }\end{array}$ & $\begin{array}{l}\text { Durch höhere Autonomie in der Arbeitserledigung kann den } \\
\text { Mitarbeitern eine höhere Freiheit geboten werden komplexe } \\
\text { Aufgaben auszuführen. }\end{array}$ \\
\hline $\begin{array}{l}\text { Reaktion auf } \\
\text { Veränderung }\end{array}$ & $\begin{array}{l}\text { Durch eine Lockerung der Rahmenbedingungen können Pilotteams in } \\
\text { dynamischen Projekten effizienter auf Änderungen reagieren. }\end{array}$ \\
\hline $\begin{array}{l}\text { Höhere Kunden- } \\
\text { zufriedenheit }\end{array}$ & $\begin{array}{l}\text { Durch einen direkten Kundenkontakt können Anforderungen } \\
\text { schneller evaluiert und Änderungswünsche mit den Kunden schnell } \\
\text { abgestimmt werden. }\end{array}$ \\
\hline
\end{tabular}

agieren. Der Teamleiter unterstützt die jeweiligen Sprecher und vernetzt diese untereinander.

Unterstützt wurden die Mitarbeiter mithilfe von agiler Software wie Jira und einem Chat-System sowie einer Filesharing Lösung, welche die schnelle und standortübergreifende Vernetzung fördert. Vor allem die Einführung und die Adaption neuer Technologie sind seit Einführung der Pilotteams sehr einfach geworden. Auch ist das Konzept sehr weit skalierbar auf viele Mitarbeiter und wechselnde Markttrends sowie Kunden.

Weiterhin wurden für die schnelle Bereitstellung von IT-Systemen und Produkten zwei weitere Teams gegründet. Klassische Standardprodukte des Unternehmens werden durch ein Produktteam (5 Mitarbeiter) nach Kanban bereitgestellt. Wohingegen individuelle und große Systeme durch ein Projektteam (15 Mitarbeiter) bereitgestellt werden. Das Team 3rd Level Operations (10 Mitarbeiter) stellt Experten für komplexe Probleme zur Verfügung und unterstützt die Teams in der Abteilung ebenfalls im Tagesgeschäft.

Insgesamt konnte das Unternehmen so für jeden Mitarbeiter von klassisch bis agil ein passendes Team zu Verfügung stellen und Kundenaufträge je nach Komplexität mit der passenden Methode erfolgreich durchführen.

\section{Fazit: Handlungsempfehlungen zur Steigerung von Agilität}

Dieser Beitrag widmet sich der Frage: Ist Agilität eine Voraussetzung oder eine Folge der Digitalisierung? Es zeigt sich, dass die Digitalisierungsprojekte oft eine erhöhte Komplexität besitzen und bei deren Umsetzung agile Methoden helfen können.

Die Teilnehmer konzipierten in der Gruppendiskussion konkrete Handlungsempfehlungen in Form eines Modells, welches das Unternehmen systematisch und selbstorganisiert in kleine Einheiten teilt. Die Einteilung wird je nach Grad der notwendigen Agilität vorgenommen. So entstehen verschiedene Bereiche mit unterschiedlicher Agilität und geringer Hierarchiestufe. Das Modell kann als Handlungsempfehlung gesehen werden, um ein Unternehmen im Sinne der Digitalisierung agil zu wandeln. Aus der Gruppendiskussion konnten die folgende Handlungsempfehlungen in Bild 4 abgeleitet werden.

\section{Schlüsselwörter:}

Agilität, Digitalisierung, KMU, Management, Mittelstand 